\title{
A service evaluation of a Hospital In-reach Service for people who are homeless in South England
}

Georgia Blackwell-Green, Dr Christopher Sargeant, Dr Priya Paudyal

Background: Homelessness has increased dramatically in the last decade, with an estimated $165 \%$ increase in rough sleeping alone in England since 2010. People experiencing homelessness can often have what is referred to as the tri-morbidity; mental health, substance and or alcohol misuse and physical health problems. Hospital admission presents a potential point of intervention to address the health problems people have been experiencing, to link them in with services and as a bridge to housing. This service evaluation explored the role of a Hospital In-reach Service team for safer hospital discharge.

Methods: A qualitative in-depth interview study was conducted with six participants related to a Hospital In-reach Service team in South England. The interviews were transcribed, and analysed following Burnard's fourteen stage method. The ethical approval was obtained from Brighton and Sussex Medical School Research Governance and Ethics Committee (ER/BSMS4284/2).

Results: Three key themes were identified; the role of the Hospital In-reach Service team, barriers to, and facilitators for advocating patients' needs. The results indicate that the service team works incredibly well, building strong and trusting relationships with patients, community and hospital teams, taking a holistic approach to patient care. However, the structural discrimination against this patient group is reiterated in this study. Despite progress, there are still problems with non-specialist staff understanding this patient group's needs.

Conclusion: There is a need for clarification within Adult Social Care regarding the distribution of responsibility between hospital and community social work teams. There also appears to be a need for expansion of training for non-specialist service staff, including ward staff, discharge teams and social workers. In-reach housing services and expansion of the service team would be invaluable.

Key Message: Hospital In-reach Service team in South England works incredibly well taking a holistic approach to patient care. Clarification in role of hospital and community social work teams is required for better management of this issue. 